\title{
Electro Dynamo Theory of Gravity (g)
}

\author{
Greg Poole \\ Industrial Tests, Inc., Rocklin, CA, USA \\ Email: greg@indtest.com
}

How to cite this paper: Poole, G. (2019) Electro Dynamo Theory of Gravity (g). Journal of High Energy Physics, Gravitation and Cosmology, 5, 773-789. https://doi.org/10.4236/jhepgc.2019.53040

Received: May 21, 2019

Accepted: June 18, 2019

Published: June 21, 2019

Copyright $\odot 2019$ by author(s) and Scientific Research Publishing Inc. This work is licensed under the Creative Commons Attribution International License (CC BY 4.0).

http://creativecommons.org/licenses/by/4.0/

\begin{abstract}
In this paper, flux transfer events from the Sun to the Earth are represented as an electrical frequency. The Earth's inner core is modeled as a motor armature and the mechanical speed is calculated from the frequency of flux transfer events. The speed of the inner core creates a centripetal acceleration at the center of the Earth that is much higher than ever thought possible. The synchronous speed is the speed of the electromagnetic field that orbits or rotates around the Earth. All electrical machines have a rotating electromagnetic field that establishes the synchronous speed. Gravity is a centripetal acceleration derived from the synchronous speed of the electromagnetic field. The mechanical speed is somewhat less based on slip, which ranges from $1 \%$ to $10 \%$ for the electrical machine we call Earth. Flux transfer event from the Sun to the Earth is what powers the machine. By adjusting for the altitude distance, from the inner core to the surface of the Earth, a value of $9.806 \mathrm{~m} / \mathrm{s}^{2}$ is calculated. The very nature of gravity has been discovered and explained using electrical equations and classical physics. There are only three forces of nature: electromagnetic, strong nuclear and weak nuclear.
\end{abstract}

\section{Keywords}

Centripetal Acceleration, Dynamo, Flux Transfer Event, Gravity (g), Gyroscope, Synchronous Speed

\section{Introduction}

Dr. Walter Elsasser, expanded on the work of Sir Joseph Larmor to advance the dynamo theory. Elsasser was the first to describe and picture the Earth as a Faraday disc: a most basic electric motor. I shall attempt to build on their work, as well as my own, and advance the electrodynamic theory of gravity [1].

This paper explores Earth's iron core acting as a constant speed armature based on a solar system's flux transfer event period of approximately every 7.1 minutes. Flux transfer events were originally reported to occur every 8 minutes 
or so according to Lockwood and Wild in their study of 1993 [2]. In 2016, Milan et al. revised the periodicity to approximately 7 to 8 minutes based on the two studies they conducted [3] [4] [5].

A 7.1-minute period equates to a frequency of $1 / 7.1 \mathrm{~min} \times 60=0.002333 \mathrm{~Hz}$. It will be readily apparent why I chose 7.1 minutes for my starting point and modeling. The distance between the Earth and the Sun varies from 146 to 152 million kilometers depending on its orbit. The inner core radius of the Earth is reported to be $1.22 \times 10^{6}$ meters [6].

Modeling techniques are revisited to construct an improved electrical drawing of the inner core. To understand the electrodynamic theory of gravity one must understand slip. Slip is the difference between synchronous or orbital speed and armature speed. Motor slip is used to explain the ranges found in 7- to 8-minute flux transfer events. Flux transfer events from the Sun create the electromagnetic field of the Earth and turn the inner core at rated armature speed. Gravity (g) is concluded to be a centripetal acceleration derived from orbital speed of the electromagnetic field. The Earth is an electrical machine and gravity is the same phenomenon that centers a rotor shaft here on Earth. Nothing is what it was. The Earth's rotor just shifted, and I will attempt to explain why it slipped. I will explain how slip and gravity are related.

\section{Earth Electrical Model Revisited}

In previous work the earth was modeled as a simple machine to better comprehend how the Earth mimics an electrical motor [7]. Briefly we determined how the circuit of the planet may be connected and discussed how the rotational speed of the outer Earth is maintained at $465 \mathrm{~m} / \mathrm{s}$. A view of the earlier model is shown below to reacquaint the reader with the electrical schematic and the context of this paper. I draw attention to the armature circuit in the center of the Earth's iron core and the armature winding $\mathrm{R}_{\mathrm{a}}$ shown as a toroid. The calculation of the synchronous speed of the magnetic field and the mechanical speed of the armature is the focus of this manuscript.

In Figure 1, An alternating current (AC) motor consists of an armature and stator, and the interaction of the currents flowing in the armature and the rotating magnetic field in the stator generates the torque that turns the shaft or in our case the iron inner core. In normal operation, with a load, the rotor speed always lags the magnetic field's speed allowing the armature to cut magnetic lines of force and produce torque. The difference between the synchronous speed of the electric motor's magnetic field, and the shaft rotating speed is slip-measured in RPM. Slip increases with increasing load-providing greater torque. Slip can be calculated from RPM or frequency. A change in speed or change in frequency has a direct proportional effect on motor slip.

\section{Calculate the Speed of Armature Using Flux Transfer Frequency}

The formula to make this calculation is the number of cycles $(\mathrm{Hz})$ times 60 (for 


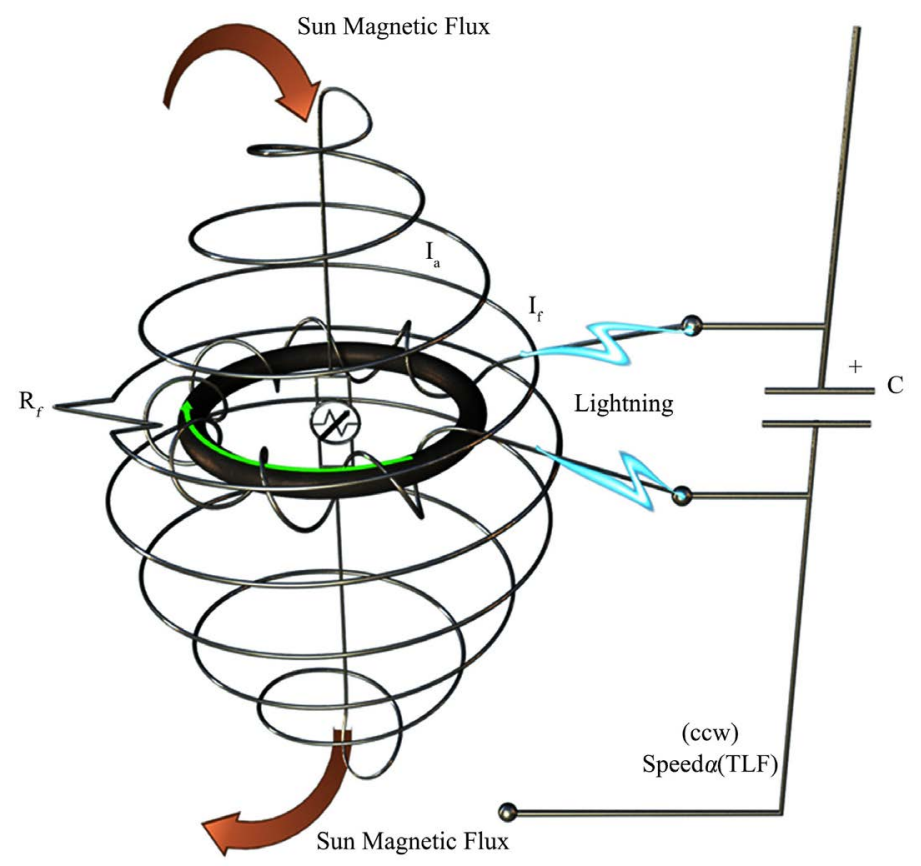

Figure 1. Electrical Schematic of Earth.

seconds in a minute) times two (for the positive and negative pulses in the cycle) divided by the number of poles. Calculating the magnetic field speed for an armature of a synchronous machine is dictated by the following equation.

$$
N=\frac{120 f}{P}[8]
$$

where,

$f=$ frequency of the iron core armature at $0.0023333 \mathrm{~Hz}$

$P=$ number of poles, or 2 .

Inserting the value for frequency and number of poles I obtain. $N=0.14$ RPM.

Below is a drawing that provides a glimpse of how the electromagnetic fields in Figure 2 are surrounding and spinning around the Earth at a higher speed than the armature. The Earth acts as a motor/generator creating its electromagnetic field from a rotating armature powered by the flux transfer of energy from the Sun.

\section{Convert Earth Armature RPM to Linear Velocity (m/s)}

RPM stands for rotations per minute and is used to quantify the speed at which an object spins, such as an electric motor or a centrifuge. Linear speed measures the actual distance travelled in meters per second. Because a rotation always covers the same distance, I can convert from rpm to linear distance using the following equation:

The RPM to Linear Velocity formula is:

$$
v=\frac{r 2 \pi \mathrm{RPM}}{60}
$$




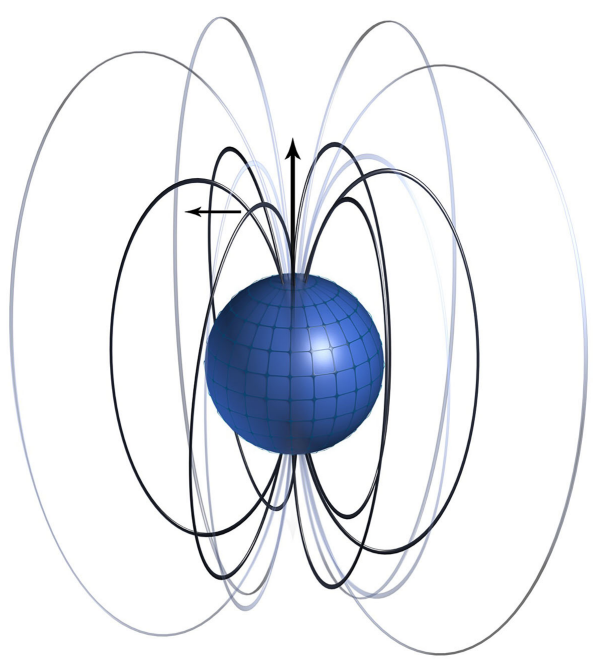

Figure 2. Earth's electromagnetic field rotating at 0.14 RPM.

or

$$
v=r \times \mathrm{RPM} \times 0.10472
$$

where:

$V=$ near velocity in $\mathrm{m} / \mathrm{s}$

$r=$ radius in meters, which for the inner iron core is $1,220,000 \mathrm{~m}$

$\mathrm{RPM}=$ Angular velocity, which is $0.14 \mathrm{RPM}$

Inserting these values, the linear velocity is calculated as $17,886 \mathrm{~m} / \mathrm{s}$.

This value is known as the tangential velocity in a centripetal configuration. Tangential velocity is the result of circular motion. Tangential velocity is a result, not a pre-requisite. It is a function of the radius of the armature and the number of rotations it makes per unit time. The larger the machine radius, the longer the distance, which must be covered during each rotation.

\section{Calculate Orbital Velocity of Inner Core}

Orbital velocity is the minimum velocity necessary to keep the object from falling into the planet. Orbital velocity generally is the pre-requisite velocity necessary for an object to stay in orbit. The shorter the radius of orbit, the closer the object is to the planet which it orbits, and the greater the gravitational attraction (centripetal attraction), which must be overcome for it to remain in orbit. So, a shorter radius means a greater velocity is required. A longer radius puts the object farther out in space and requires less velocity to keep it from falling into the planet. So, a longer radius means less velocity is needed.

When one of the masses is almost negligible compared to the other mass, as is the case for inner iron core and its orbiting electromagnetic field, one can approximate the orbital velocity as:

$$
V=\sqrt{\frac{G M}{r}} \quad[10]
$$

where, 


$$
\begin{aligned}
& \text { Newton's Constant }=G=6.674 \times 10^{-11} \\
& \text { Mass of the Earth }=M=5.972 \times 10^{24} \mathrm{~kg} \\
& \text { Radius of inner core }=r=1.22 \times 10^{6} \mathrm{~m}
\end{aligned}
$$

Inserting the values, I get a result of $18,077 \mathrm{~m} / \mathrm{s}$.

\section{Calculate Acceleration at the Inner Core}

The centripetal ("center-seeking") acceleration is the motion inwards towards the center of a circle. The acceleration is equal to the square of the velocity, divided by the radius of the circular path.

$$
a=\frac{v^{2}}{r} \quad[11]
$$

where,

$$
\begin{aligned}
& a=\text { acceleration inward } \\
& V=\text { velocity }=18,075 \mathrm{~m} / \mathrm{s}
\end{aligned}
$$

Radius of inner core $=r=1.22 \times 10^{6} \mathrm{~m}$

When we insert these values in the centripetal acceleration equation, we obtain a result of $268 \mathrm{~m} / \mathrm{s}^{2}$, which represents the gravity, or centripetal acceleration at the surface of Earth's inner core in Figure 3.

\section{Calculate Acceleration by Altitude (m/s $)$}

The acceleration due to gravity by altitude formula computes the approximate acceleration due to gravity on the surface of the Earth based on the altitude [12]. In this paper, I use this equation to calculate the specific altitude above the inner iron core, where an object is at the surface of the Earth. The altitude $(h)$ is then the radius of the earth less the radius of the inner core.

This same equation applies to the electromagnetic field whereby intensity is inversely proportional to the inverse square of a wire conductor which represents the Earth's armature [13]. The equation for the acceleration due to gravity based on altitude is:

$$
g_{\text {alt }}=g\left(\frac{r_{e}}{r_{e}}+h\right)^{2}
$$

where:

$g_{\text {alt }}=$ the acceleration due to gravity at a specific altitude

$r_{e}=$ Mean Radius of the Iron Core: $1.22 \times 10^{6} \mathrm{~m}$

$g=$ Acceleration due to gravity at the Iron Core: $268 \mathrm{~m} / \mathrm{s}^{2}$

$h=$ Altitude above the Iron Core: $6.378 \times 10^{6}-1.22 \times 10^{6}=5.158 \times 10^{6} \mathrm{~m}$.

Inserting the values into the equation, we obtain a result of $9.807 \mathrm{~m} / \mathrm{s}^{2}$ or $\mathrm{g}$ at the surface of the Earth. The square root of this number divided by the radius of the Earth is the orbital velocity at the Earth's surface, which equates to $7910 \mathrm{~m} / \mathrm{s}$. Since the magnetic field intensity is proportional to $1 / r^{2}$ this number also represents the orbital speed of the electromagnetic field as it is rotating around the surface of the Earth. 


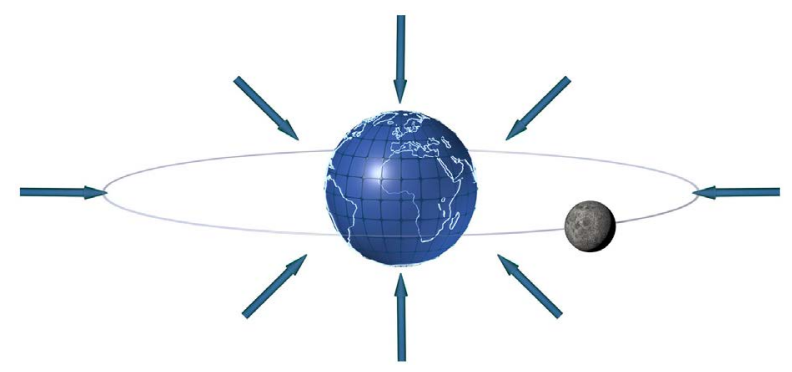

Figure 3. Centripetal Force.

\section{Calculate Magnetic Field and Primary Current of Inner Core}

To calculate the magnetic field and primary current of the inner core, I use the formula to calculate the field at the center of a current loop as described below in Figure 4.

The form of the magnetic field from a current element in the Biot-Savart law becomes:

$$
\mathrm{d} B=\frac{\mu_{0} I \overrightarrow{\mathrm{d} L} \times \overrightarrow{\hat{r}}}{4 \pi R^{2}}=\frac{\mu_{0} I \mathrm{~d} L \sin \theta}{4 \pi R^{2}}
$$

which in this case simplifies greatly because the angle equals 900 for all points along the path and the distance to the field point is constant. The integral becomes

$$
\begin{gathered}
B=\frac{\mu_{0} I}{4 \pi R^{2}} \oint \mathrm{d} L=\frac{\mu_{0} I}{4 \pi R^{2}} 2 \pi R=\frac{\mu_{0} I}{2 R} \\
\mu_{0}=4 \pi \times 10^{-7} \mathrm{~T} \cdot \mathrm{m} / \mathrm{A}
\end{gathered}
$$

In this special case the symmetry is such that the field contributions of all the current elements around the circumference add directly at the center. The line integral of the length is just the circumference of the circle.

Using a current of approximately $1.373 \times 10^{10} \mathrm{~A}$ the center of the Earth has a field strength of roughly 70 Gauss and the field at the surface is calculated as 0.46 Gauss. The range of the magnetic field at the surface of the Earth is 0.22 to 0.65 gauss.

\section{Calculate the Field at the Surface of the Inner Core}

Using the same formula as in the previous section, I now calculate the field at the surface of the inner core. I inserted the distance $\mathrm{Z}$ to the edge of the inner core, into the formula, and the result is 25 Gauss which matches the published measured value of 25 Gauss [14].

\section{Magnetic Force within the Earth's Inner Core}

To determine the magnetic force within the Earth's inner core, I compare it to a scenario of a positive charge moving through a stationary wire in a magnetic field (Figure 5). Here the magnetic force is given by: 


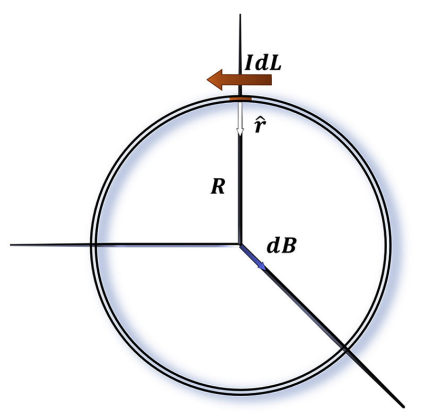

Figure 4. Field at the centre of a current loop.

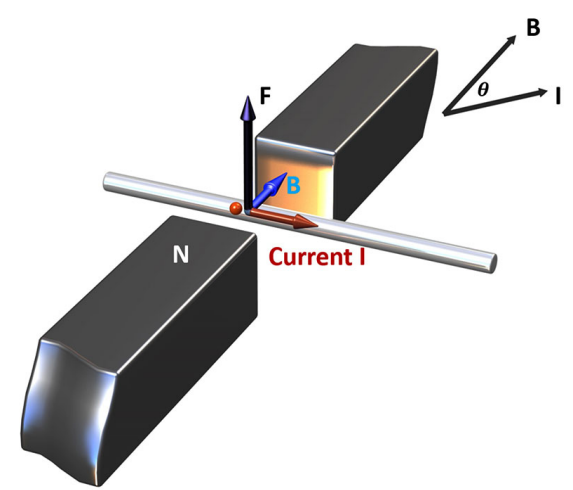

Figure 5. Positive charge moving through a stationary wire in a magnetic field.

$$
F=q v B \sin \theta
$$

which for a charge $q$ travelling length $L$ in a wire can be written as:

$$
\begin{gathered}
F=q \frac{L}{t} B \sin \theta \\
F=\frac{q}{t} L B \sin \theta \\
F=I L B \sin \theta
\end{gathered}
$$

The magnetic force on a current-carrying wire is perpendicular to both the wire and the magnetic field with the direction given by the right-hand rule (Figure 6). According to the rule, curl the fingers as if rotating vector $I$ into vector $B$, the thumb is then in the direction of the force $F$. Since the angle is 90 degrees the equation $F=I L B \sin \theta$ becomes:

$$
\begin{gathered}
F=I L B \sin 90 \\
F=I L B
\end{gathered}
$$

Inserting the following values into the above equation: current of $1.373 \times 10^{10}$ A, the circumference of the inner core of $7.7 \times 10^{6} \mathrm{~m}$, angle of 40 degrees and a magnetic field of 25 Gauss results in a force of $1.69118 \times 10^{14} \mathrm{~N}$.

\section{Inner Core Torque Calculation}

I now calculate the torque of the inner core in the following manner. Figure 7 


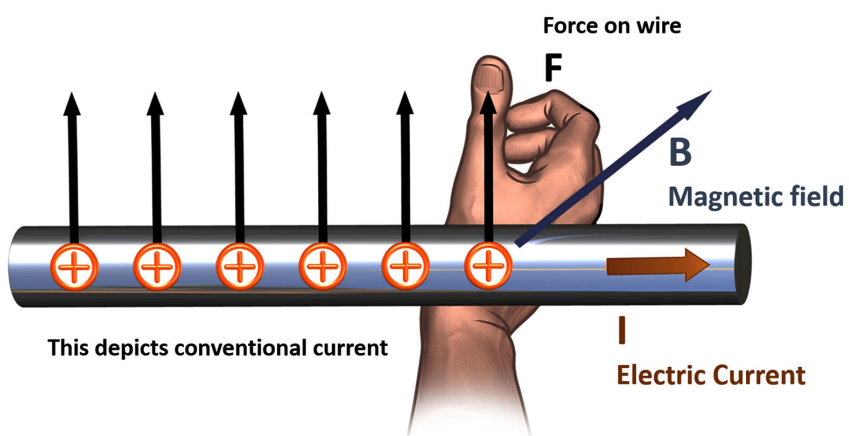

Figure 6. The magnetic force on a current-carrying wire is perpendicular to both the wire and the magnetic field with the direction given by the right-hand rule.

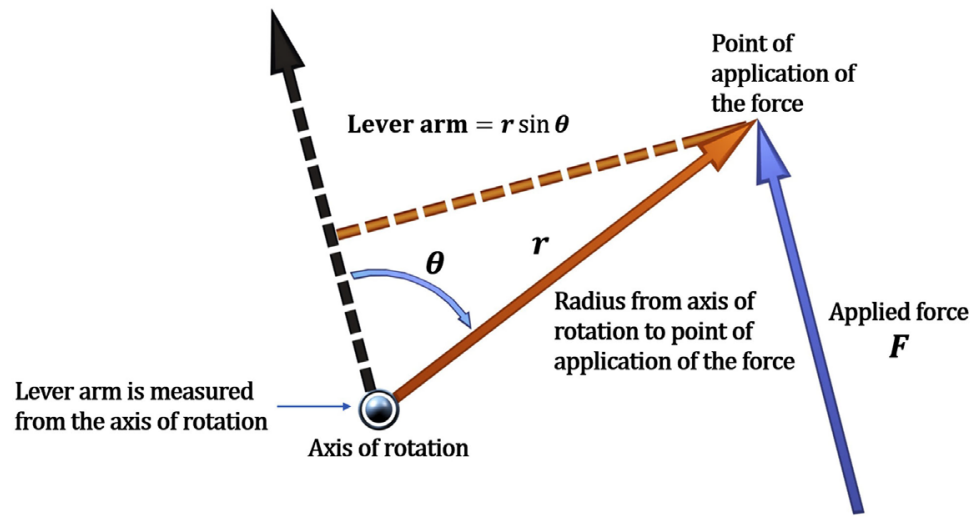

Figure 7. Torque is calculated using the angle between the $\mathrm{r}$ and $\mathrm{F}$ vectors.

illustrates the method of calculating torque around an axis of rotation, which is dependent on the force $(F)$ applied, the radius $(r)$ from the axis of rotation to the point of application of the force and the angle $(\theta)$, which is $\leq 180$ degrees between the $r$ and $F$ vectors when they are drawn from the same origin. The direction of the torque is given by the right-hand rule, which gives a vector out towards the reader in this case. Note that the torque is maximum when the angle is 90 degrees. Torque is calculated using the following equation:

$$
\text { Torque }=\tau=r F \sin \theta
$$

A practical way to calculate the magnitude of the torque is to first determine the lever arm and then multiply it times the applied force. The lever arm is the perpendicular distance from the axis of rotation to the line of action of the force.

Inserting force of $1.69 \times 10^{14} \mathrm{~N}$, an angle of 78.50 and the radius of the inner core $\left(1.22 \times 10^{6} \mathrm{~m}\right)$ gives us a torque of $2.02172 \times 10^{20} \mathrm{Nm}$. I note that the lever arm is slightly inward from the surface of the inner core. The angle of 78.50 reflects the magnetic tilt of the Earth at 11.5 degrees.

\section{Calculate Torque and Speed Constant of Inner Core}

The torque and speed of the inner core can be calculated using the following formula: 


$$
\begin{gathered}
K_{t}=\frac{\text { Torque }}{\text { Current }} \\
K_{t}=\frac{2.0217 \times 10^{24}}{1.373 \times 10^{10}} \\
K_{t}=4.73 \times 10^{9} \\
K_{s}=\frac{1}{4.73 \times 10^{9}} \\
K_{s}=6.79 \times 10^{-11} \text { or Newton's Constant }
\end{gathered}
$$

Thus, the error in calculating Newton's Constant is less than $1 \%$.

\section{Voltage of the Earth's Inner No Load}

When a conductor moves through a magnetic field, there will be a generated motional emf. This is one example of Faraday's law and it arises from the magnetic force (Figure 8). The voltage generated in a length of wire, presuming the entire length moves through a uniform field is given below:

$$
e m f=v B L \sin \theta
$$

If $\theta=90^{\circ}$

$$
e m f=v B L
$$

If the velocity is perpendicular to the magnetic field, then the generated voltage is given by the simple product:

$$
\text { Generated voltage }=e m f=\text { Volcity } \times B \text { field } \times \text { Length }
$$

Using the circumference of the Earth, velocity at synchronous speed (orbital speed), an angle of $40^{\circ}$, and a magnetic field of 25 Gauss, the result is $2.22 \times 10^{8}$ $\mathrm{V}$. When unloaded a machine speed will approach that of the speed of the electromagnetic field and produce full voltage. In our example, the voltage and current are in phase, or unity power factor. The Earth has a large power factor correction capacitor in the atmosphere.

\section{Calculate Voltage Constant and Speed Constant}

I calculate the voltage and speed constant to better understand the electrical characteristics of the machine we call Earth. Torque, voltage and speed constants are common values that electrical engineers use to size and apply motors to loads. For the purpose of this paper, however, I am trying to get a feel as to the magnitude of the voltages and currents within the inner core. I also want to try and understand how the inner core winding or current path is pitched, for future gyroscopic simulation. Typically, motor and generator coils are pitched at different angles for a variety of mechanical reasons that we can research and discuss in future papers. Assuming Newton's constant is truly constant in the future I can use these basic motor characteristic equations to change pitch angles and see what effects they have on the machine. The voltage constant $k_{V}$ can be calculated using the following formula: 


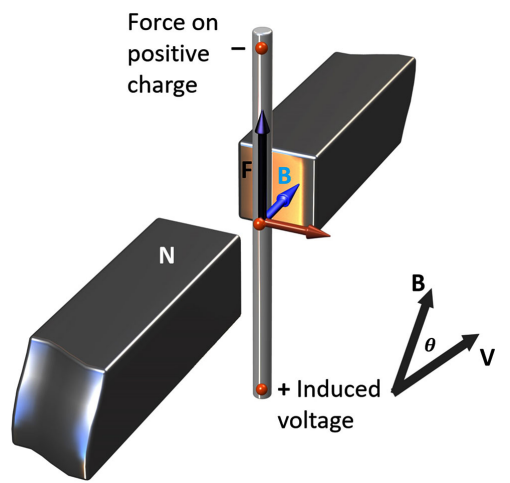

Figure 8. A motional emf is generated when a conductor, such as a wire, is moved thorough a magnetic field.

$$
k_{v}=\frac{\text { Voltage }}{\omega(\mathrm{rad} / \mathrm{s})}
$$

where,

$$
\begin{gathered}
V=2.22 \times 10^{8} \mathrm{~V} \\
\omega=18075 \mathrm{~m} / \mathrm{s}=0.0148155(\mathrm{rad} / \mathrm{s}) \\
k_{v}=1.5 \times 10^{10}
\end{gathered}
$$

The speed constant is a reciprocal of the voltage constant.

$$
\begin{gathered}
k_{s}=\frac{1}{k_{v}}=\frac{1}{1.5} \times 10^{10} \\
k_{s}=6.68 \times 10^{-11}
\end{gathered}
$$

This value is approximately equal to Newton's Constant, which is $6.674 \times$ $10^{-11} \mathrm{~m}^{3} \cdot \mathrm{kg}^{-1} \cdot \mathrm{s}^{-2}$

\section{Voltage of Inner Earth at Full Load}

In this example, I show the typical running condition of the Earth where the inner core velocity decreases to our previously calculated $17,886 \mathrm{~m} / \mathrm{s}$ based on 7.1 minutes flux transfer events. As the load increases, speed and frequency will decrease and torque will increase. Voltage of the inner core drops to 220.3 million volts in this example. Normal running current was shown previously at approximately $1.73 \times 10^{10}$ Amps. Assuming unity power factor the inner core consumes roughly $3.08 \times 10^{18} \mathrm{~W}$. I note this number nearly matches my previous calculation of $3.14 \times 10^{18}$ watts in my previous paper [15].

$$
K_{s}=\frac{1}{K_{v}}=\frac{1}{\frac{220309330}{0.01466 \mathrm{rad} / \mathrm{s}}}=6.65 \times 10^{-11} \text { (Newton's Constant) }
$$

\section{Calculate Centripetal Acceleration from Gravitational Parameters}

As a way of checking my result I will use the Earth's standard gravitational pa- 
rameter $(\mu)$

to derive centripetal acceleration at a synchronous speed of the electromagnetic field.

$$
\mu=r v^{2}
$$

where,

$$
\begin{gathered}
r=1.22 \times 10^{6} \mathrm{~m} \text { (Inner Core Radius) } \\
\mu=3.986 \times 10^{14} \mathrm{~m}^{3} / \mathrm{s}^{2}
\end{gathered}
$$

Inserting the values and the result is $v=18075 \mathrm{~m} / \mathrm{s}$.

Inserting the velocity into the centripetal equation I obtain the following:

$$
\begin{gathered}
a=\frac{v^{2}}{r} \\
a=268 \mathrm{~m} / \mathrm{s}^{2}
\end{gathered}
$$

Inserting the acceleration of the inner core into the Altitude Equation

$$
\begin{gathered}
g=268 \times\left(\frac{1.22 \times 10^{6}}{6.376 \times 10^{6}}\right)^{2} \\
g=9.806 \mathrm{~m} / \mathrm{s}^{2}
\end{gathered}
$$

\section{Model the Toroid Armature}

Finding the magnetic field inside a toroid is a good example of the power of Ampere's law. In Figure 9, the current enclosed by the blue line is just the number of loops times the current in each loop. Ampere's law then gives the magnetic field by

$$
\begin{gathered}
B 2 \pi r=\mu N I \\
B=\frac{\mu N I}{2 \pi r}
\end{gathered}
$$

The toroid is a useful device used in everything from tape heads to tokamaks.

Magnetic field $=$ permeability $\times$ turn density $\times$ current

Inserting the radius, 0.005 turns, $1.466 \times 10^{10} \mathrm{amp}$ and I obtain a magnetic field of 24 gauss. Professor Eric Laithwaite of Imperial College London put together this video "The Circle of Magnetism" in 1968 [16]. Towards the end he suspends a small sphere and then a larger sphere using an electrical coil. He asserts that that a second coil is needed to center the sphere (Figure 10). This leads me to believe that the iron inner core is held in place by a top and bottom coil which I will now show below as two separate toroids.

\section{Calculate Centripetal Acceleration $\left(\mathrm{m} / \mathrm{s}^{2}\right)$ of the Earth's Mantle}

The acceleration is equal to the square of the velocity, divided by the radius of the circular path. 


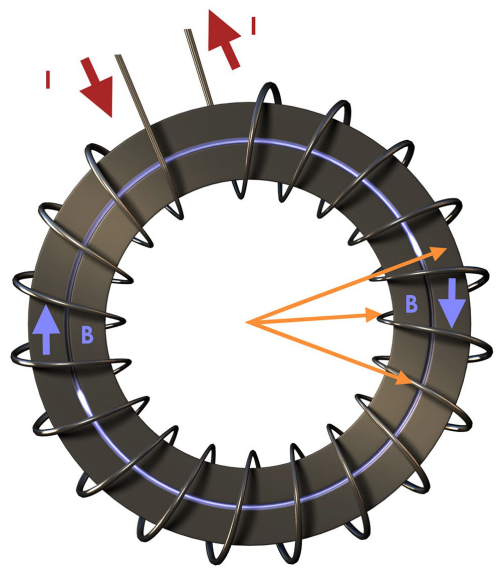

Figure 9. Magnetic field of a toroid.

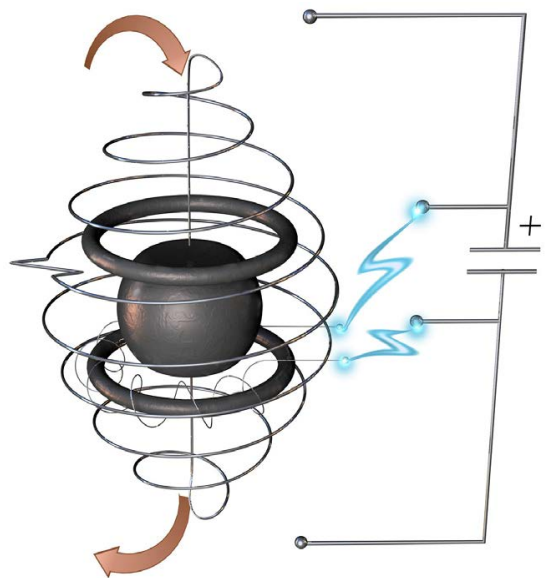

Figure 10. The Electrical Earth with two inner holding coils and a nuclear flux ball armature.

$$
a=\frac{v^{2}}{r}
$$

where,

$$
\begin{aligned}
& a=\text { acceleration inward } \\
& V=\text { velocity, or } 465 \mathrm{~m} / \mathrm{s} \\
& r=\text { radius of inner core or } 6.378 \times 10^{6} \mathrm{~m}
\end{aligned}
$$

When we insert these values in our centripetal acceleration equation, we obtain a result of $0.034 \mathrm{~m} / \mathrm{s}^{2}$. The rotational speed of the outer Earth mantle or shunt field is not a significant contributor to gravity at less than $0.3 \%$. The outer mantel acts as the spin axis of a gyroscope. Gyroscopes are used to maintain orientation and angular velocity.

\section{Calculate Slip of Earth}

The AC induction motor slip formula is [17]:

$$
\text { slip }=\frac{n_{s}-n}{n_{s}} \times 100
$$


where,

$$
\begin{aligned}
& n_{s}(\text { synchronous speed })=18,075 \mathrm{~m} / \mathrm{s} \\
& n(\text { rotational speed in } \mathrm{rpm})=17,886 \mathrm{~m} / \mathrm{s}
\end{aligned}
$$

The slip of the Earth is calculated to be $1.0 \%$ at 7.1 minutes. Increasing the time period between flux transfer events from 7.1 minutes to 7.5 minutes will raise the slip of the Earth to 5\%. At 8 minutes the Earth slip will approach 11\%. Typical motor slips for earthly electrical machines is $1 \%$ for large horsepower motors and up to $5 \%$ for fractional motors.

Based on the reported time periods the frequency will vary from 0.00208 $0.00238 \mathrm{~Hz}$. Speeds as low as $16,000 \mathrm{rpm}$ to as high as $18,000 \mathrm{rpm}$ will result. The Earth should never run at synchronous speed in theory. The inner core will expand and contract as electrical load increases and decreases. Ranges of the inner core radius vary from $1100 \mathrm{~km}$ to $1400 \mathrm{~km}$ based on seismographs. The wavelength and frequency also change with seasonal orbits as the distance from the Earth to the Sun change.

Since the Earth and the Sun act as an electrical power system we can conclude that changes in the Sun's power output will have an effect on the Earth's frequency and slip. Likewise load changes that occur at the Earth will be seen by the Sun. Voltage and current swings will be seen on both ends of the wireless transmission line. The Sun as a transmitter is tied to the Earth as a receiver through flux transfer events. The Sun acts as a cosmic power generator and the Earth as a planetary motor load. It is reasonable to expect that watts, vars and kVA are exchanged via the wireless transmission system just like a power grid.

\section{Conclusions}

My calculations demonstrate that the rotation of the Earth's armature circuit is the source of gravity that we experience on the surface of the Earth. Gravity is a centripetal acceleration derived from the inner iron core rotation, which acts as a motor armature. The flux transfer frequency of the cosmic solar system establishes the frequency of the armature. Using a period of around 7.1 minutes between flux transfer events gives us a frequency of approximately $0.00233 \mathrm{~Hz}$. Like any motor here on Earth the frequency of the system establishes the rate running speed of the machine. The synchronous speed of the electromagnetic field matches that of the orbital velocity of electromagnetic field. Our earthly armature travels at a speed of 0.14 RPM. When converted to velocity, I calculate a centripetal acceleration at the surface of the inner iron core of $268 \mathrm{~m} / \mathrm{s}^{2}$. Adjusting for the distance or altitude from the inner core to the surface of the Earth, we obtain a result of $9.807 \mathrm{~m} / \mathrm{s}^{2}$ which matches gravity. This number in turn corresponds to a lineal velocity of $7910 \mathrm{~m} / \mathrm{s}$, which represents the orbital speed of the electromagnetic field at the surface of the Earth. This figure of 7910 $\mathrm{m} / \mathrm{s}$ is already known as the orbital speed at the surface of the Earth. This finding confirms my first paper where the electrodynamic theory of gravity was first proposed [18]. Field test data on the velocity of the Earth's electromagnetic field 
at the surface of the Earth will prove the theory beyond question. Understanding the workings of the inner core of the Earth is also paramount.

Orbital speed is equivalent to synchronous speed of the Earth's electromagnetic field. The synchronous speed less the mechanical speed of the armature is commonly referred to as motor slip. The Earth has a slip of approximately $1 \%$, which is similar to electrical motors on Earth. Gravity is based on the orbital speed of the electromagnetic field, which will always be slightly higher than the actual speed of the armature due to load conditions of the inner core.

I have used 25 Gauss for the surface of the inner core and a radius of $1.22 \times$ $10^{6} \mathrm{~m}$ as my starting point. This equates to the center of the Earth having a magnetic field of 70 Gauss and an outer surface magnetic field of 0.46 Gauss. An inner core current of $6.5 \times 10^{6} \mathrm{~A}$ will result in a minimum field at the surface of the Earth of 0.22 Gauss and 33 Gauss in the center of the Earth using the Biot-Savart model. Creating a peak surface magnetic field of 0.65 Gauss will result in the center of the Earth rising to 97 Gauss; with a peak current of $1.9 \times 10^{10} \mathrm{~A}$. The peak in flux may be related to solar phenomena, which may cause a spike in the magnitude of flux transfer events. I conclude that changes in magnetic field on the surface of the Earth are the result of changes in magnitude and periodicity of flux transfer events, which cause the primary current of the inner core to fluctuate up and down between roughly 6 and $20 \times 10^{9} \mathrm{~A}$.

Knowing that the inner core expands and contracts I deduce that currents are not uniform throughout the core. Operating frequency also changes with orbits as the distance from the Sun increases and decreases. During summer periods, frequency will be higher, while it will be lower in winter. The inner core will expand and contract as frequency changes from orbits, load, and flux transfer events change. The inner core is very dynamic and has a myriad of internal and external influences that dictate its physical behavior.

The magnitude of flux during flux transfer events range from approximately 50 to $100 \mathrm{MWb}$ [3]. If I divide $1 \times 10^{8} \mathrm{~Wb}$ by the area of the inner core $4.68 \times$ $10^{12} \mathrm{~m}^{2}$, the result is 0.00002 Weber per square meter, or 0.2 Gauss-not sufficient to power our Earth. However, it has been reported by C.T. Russell and R.C. Elphic that in the vicinity of the magnetopause when the magneto sheath field is southward there is reconnection, and the flux transfer rates for these events are on the order of 1 to $2 \times 10^{12}$ Maxwell's per second, or more [19]. Dividing this range of peak values by the area of the inner core results in 21.3 to 42.7 Gauss. These values are in the range of those calculated in this paper. Additional research is required to establish flux transfer RMS and detailed positive and negative peaks, and harmonics, of Flux Transfer Events.

The Earth is a gyroscope with a spinning inner core and a counter spinning mantle. The velocity of the outer mantle is $465 \mathrm{~m} / \mathrm{s}$ at a magnetic 11.5 degrees. The inner solid core spins in the opposite direction with a tilted magnetic 40 degrees. The Earth's gyroscope has at least two wire wound coils or gimbles and may have a third if we include the liquid outer core. The electrical coils of the 
inner core and outer mantle are pitched at opposing angles to counterbalance the gyroscope and give the Earth its polar orientation and differing angular velocities.

This paper furthers our understanding of planets as electrical machines and confirms earlier work. I have proven that flux transfer events are the frequency for cosmic wireless power transfer from the Sun to the planets; that Newton's Constant is a motor speed constant; and that gravity is a centripetal acceleration derived from electromagnetism. There are only three forces of nature, gravity is not a force. There is no dedicated force or field behind gravity. Gravity in fact does not exist and never did. Gravity is a misnomer that was first used in science in the $17^{\text {th }}$ century. It has been wrongly applied to describe a centripetal acceleration. Dr. Albert Einstein correctly pointed out that gravity is an acceleration, and my research, calculations and systematic analysis have proven his claim. Einstein was accurate in his elevator thought experiment. Sir Joseph Larmor was also right about the Sun being a self-excited dynamo, and so was Sir J.J. Thomson the first to model an earthly spheroid and write tantalizing equations of force. Dr. Walter Elsasser also contributed much with his explanation of a Faraday Disc and thermodynamic modeling of Earth. However, Nickola Tesla was perhaps most correct when at 82 he made mention of an electro dynamic theory of gravity in a press conference.

The Sun acting as a generator and the Earth as a motor are as self-evident as the changing of the days. Connecting the two through cosmic wireless transmission via flux transfer events has been possible in our generation. The simple observation of a time period of 7 to 8 minutes was the catalyst for making known what has long been intuited by previous thought leaders. It is my hope that this paper will be well received, and the electrodynamic theory of gravity be studied and contemplated by my peers in electrical engineering and physics.

These monstrous machines, called planets, are ours to operate, maintain and care for. But, be careful when we tinker with electricity, for 222 million volts and 14 billion amps could be lethal to an entire civilization. Periods of 8 minutes between flux transfer events is a concern as a motor slip of $11 \%$ appears unusual from an electrical engineering standpoint. Our machine does not have rotor bars to short-out, but intuitively it seems the armature is lagging under load and falling behind the natural frequency of the electromagnetic field. Is this a normal cyclical event, or an anomaly? Has the industrial revolution and man's need for fossil fuels somehow had an impact on the speed of the armature? Are we humans' part of the $3.08 \times 10^{18} \mathrm{~W}$ energy equation? Are we loading down the armature and are we a factor in an $11 \%$ slip? These are initial, yet interesting questions that can only now be brought into the environmental discussion. Greenhouse gases are an alarming trend, but a stalled rotor of the Earth would be the end of the world as it would stop turning. All life would instantly end in one single electrifying moment if the rotor flashed over due to a short circuit. Electrical problems for large rotating apparatuses are typically catastrophic in nature. It 
behooves scientists to learn about our electrical Earth and what can go wrong should we humans interfere with the natural current flow or electrical insulation system. Finally, machines also wear out. We should do a conditional assessment and periodic testing of the Earth's electrical system. There is so much to learn about how planets are wired.

This earthly motor has run in harmony with the Sun for billions of years and let it always be so. We should not tamper or develop the magnetic polar regions, where our life-giving energy connection with the Sun passes through to the inner core. Any interference with the magnetic flux transfer is at our peril. The north and south poles need to be continually monitored. Satellite data need to be customized for better electrical analysis.

If you are moved by my concerns, if you felt the Earth beneath you suddenly slip at the release of my thoughtful energeia, do not despair, for shared knowledge of our world is the path to humanity being truly synchronized. The barmaid will know exactly what we are talking about when one of us points to the fan rotating about her head and tells her how the Earth rotates and how centripetal acceleration holds the fan together and her feet to the floor. We need to be talking electrical reluctance and electrical relevance to the masses. When everyone knows how the Earth works and understands its limitations, they will also learn what needs to be done to ensure that it continues to perform efficiently and with minimal upset and disturbance. Facts will replace passion; environmental solutions will be based on engineering calculation and well thought out plans.

And, so I offer a celebratory toast to all the great men we have read about and to the original electro dynamo theory on the year of its centennial- "God save the Queen... and the papers of Sir Joseph Larmor"!

\section{Acknowledgements}

The author wishes to acknowledge ASK Scientific (https://www.askscientific.com) for the formatting assistance and artwork. Special thanks to Clifford Mosher, college professor and academic advisor for his early instruction on rotating $\mathrm{AC} / \mathrm{DC}$ machines and electrical power systems.

\section{Conflicts of Interest}

The author declares no conflicts of interest regarding the publication of this paper.

\section{References}

[1] Elsasser, W. (1958) The Earth as a Dynamo. Scientific American, 198, 44-48. https://doi.org/10.1038/scientificamerican0558-44

[2] Lockwood, M. and Wild, M.N. (1993) On the Quasi-Periodic Nature of Magnetopause Flux Transfer Events. Journal of Geophysical Research, 98, 5935-5940. https://doi.org/10.1029/92JA02375

[3] Fear, R.C., Trenchi, L., Coxon, J.C. and Milan, S.E. (2017) How Much Flux Transfer 
Does a Flux Transfer Event Transfer. JGR Space Physics, 122, 12,310-12,327. https://doi.org/10.1002/2017JA024730

[4] Milan, S.E., Lester, M., Greenwald, R.A. and Sofko, G. (1999) The Ionospheric Signature of Transient Dayside Reconnection and the Associated Pulsed Convection Return Flow. Annales Geophysicae, 17, 1166-1171. https://doi.org/10.1007/s00585-999-1166-2

[5] Milan, S.E., Lester, M., Cowley, S.W.H. and Brittnacher, M. (2000) Convection and Auroral Response to a Southward Turning of the IMF: Polar UVI, CUTLASS, and IMAGE Signatures of Transient Magnetic Flux Transfer at the Magnetopause. Journal of Geophysical Research, 105, 15,741-15,755. https://doi.org/10.1029/2000JA900022

[6] Ahrens, T.J. (1995) Global Earth Physics A Handbook of Physical Constants. American Geophysics Union, Washington DC, 93.

[7] Poole, G. (2018) Dynamo Speed Control and Tectonics-Modeling Earth as a Shunt Wound DC Machine. Journal of High Energy Physics, Gravitation and Cosmology, 4, 152-165. https://doi.org/10.4236/jhepgc.2018.41014

[8] Brumbach, M.E. and Clade, J. (2003) Industrial Maintenance. Thomson Delmar Learning, Library of Congress, Washington DC, 400.

[9] Raymond, H.M. (1910) Cyclopedia of Mechanical Engineering: A General Reference Work on Machine Shop.... Chicago American Technical Society, Chicago, Volume 3, 10 .

[10] Seeds, M. (2009) The Solar System. Thomson Higher Education, Belmont, 83.

[11] Breithaupt, J. (2004) Physics for OCR. Nelson Thomas Ltd., Nashville.

[12] Dennis, J.T. (2003) The Complete Idiots Guide to Physics. Alpha Books, Indianapolisi, 103.

[13] Shadowitz, A. (1975) The Electromagnetic Field. McGraw-Hill Book Company, Pennsylvania, 88.

[14] Buffett, B. (2010) Tidal Dissipation and the Strength of the Earths Internal Magnetic Field. Nature, 468, 952-954. https://doi.org/10.1038/nature09643

[15] Poole, G. (2018) Cosmic Wireless Power Transfer System and the Equation for Everything $\mathrm{E}=\mathrm{mc} 2=\mathrm{vc} 2 / 60=\mathrm{a} 3 / \mathrm{T}=\mathrm{G}(\mathrm{M} 1+\mathrm{M} 2) / 4 \pi 2=(\mathrm{KE}+\mathrm{PE}) / 1.0 \mathrm{E} 15=\mathrm{Q}=$ $\mathrm{PA} / \mathrm{F}=\lambda / \mathrm{hc}=1 / 2 \mathrm{q}=\mathrm{VI}=1 / 2 \mathrm{LI} 2=1 / 2 \mathrm{CV}=\mathrm{I} 2 \mathrm{R}=\ldots$ Journal of High Energy Physics, Gravitation and Cosmology, 4, 588-650. https://doi.org/10.4236/jhepgc.2018.44036

[16] Laithwaite, E. (1968) Video: The Circle of Magnetism. Imperial College of London in Association with the Royale Institute, London.

[17] Zocholl, S. (2003) AC Motor Protection. Schweitzer Engineering Laboratories, Pullman.

[18] Poole, G. (2017) Theory of Electromagnetism and Gravity. Journal of High Energy Physics, Gravitation and Cosmology, 3, 663-692. https://doi.org/10.4236/jhepgc.2017.34051

[19] Russell, C.T. and Elphic, R.C. (1979) ISEE Observations of Flux Transfer Events at the Dayside Magnetopause. Geophysical Research Letters, 6, 33-36. https://doi.org/10.1029/GL006i001p00033 